\title{
Normal erythrocyte membrane Gs $\alpha$ bioactivity in two unrelated patients with acrodysostosis
}

\author{
L C Wilson, M E M Oude Luttikhuis, M Baraitser, H M Kingston, R C Trembath
}

\begin{abstract}
Shortening of the tubular bones of the hands and feet with cone shaped epiphyses is known as peripheral dysostosis and is common to several syndromes including acrodysostosis and Albright's hereditary osteodystrophy (AHO). The underlying defect in AHO is known to be a reduction in bioactivity of the $\alpha$ subunit of the signal transducing protein, Gs, and heterozygous deactivating mutations have been shown in the Gsa gene. Because of additional overlapping clinical and radiological features it has been suggested that acrodysostosis and AHO represent poles of a single diagnostic spectrum. We have measured Gs $\alpha$ bioactivity in two unrelated patients with a clinical diagnosis of acrodysostosis and found both to be normal. Mutation analysis of the Gsa gene showed no sequence variation in 12 of the 13 exons examined. These results indicate that, at least in a proportion of patients with acrodysostosis, the condition is aetiologically distinct from AHO.
\end{abstract}

( $($ Med Genet 1997;34:133-136)

Keywords: acrodysostosis; pseudopseudohypoparathyroidism; Gs $\alpha$; Albright's hereditary osteodystrophy.

Shortening of the tubular bones of the hands and feet with cone shaped epiphyses but without other major skeletal abnormalities was called peripheral dysostosis by Brailsford ${ }^{1}$ in 1948. Between 1963 and 1971, several authors described a distinctive syndrome characterised by severe generalised peripheral dysostosis associated with nasal hypoplasia and mental retardation. The names PNM and acrodysostosis were suggested, the latter being more widely used..$^{2-5}$ Additional findings included short stature (often of prenatal onset), relative first ray hyperplasia in the feet, loss of caudal widening of vertebral interpedicular distances, advanced skeletal maturation, increased mandibular angle, recurrent otitis media and hearing loss, but no biochemical or endocrine disturbance. ${ }^{6}$

Similar skeletal changes may occur in Albright's hereditary osteodystrophy (AHO) where they are frequently associated with short stature and developmental delay. ${ }^{7}$ The biochemical defect underlying $\mathrm{AHO}$ is a reduction in activity of the $\alpha$ subunit of a signal transducing $G$ protein, Gs, which stimulates intracellular adenylyl cyclase in response to various peptide hormones. ${ }^{8}$ Several heterozygous deactivating mutations in the Gs $\alpha$ gene on chromosome $20 \mathrm{q} 13$ have now been described. ${ }^{9}$ Patients with AHO are divided into two distinct phenotypes known as pseudohypoparathyroidism (PHP) and pseudopseudohypoparathyroidism (PPHP) on the basis of endocrine findings. In those with $\mathrm{PHP}$, resistance can be shown to various hormones which are transduced by Gs $\alpha$, most notably PTH. In contrast, no endocrine resistance is found in PPHP despite the equivalent reduction in Gs $\alpha$ bioactivity. Ectopic ossification, the basis of which is unknown, occurs frequently but not always in both forms of AHO.

Although recent molecular advances have proved the value of delineating conditions on the basis of consistently observed combinations of clinical features, in some instances closely overlapping conditions have turned out to have a common aetiology. As a result of the phenotypic overlap between acrodysostosis and PPHP, some authors have considered them part of a single disease spectrum. ${ }^{11}{ }^{11}$ To address this question we have measured Gsa bioactivity and screened for mutations in the Gs $\alpha$ gene in two unrelated children with clinical features of acrodysostosis.

\section{Case reports and methods}

CASE 1

This is the third of four children born to healthy, unrelated parents. Intrauterine growth retardation was detected prenatally and she was delivered at 38 weeks' gestation by caesarian section, weighing $2020 \mathrm{~g}$ ( $<3$ rd centile) with a head circumference of $32 \mathrm{~cm}$ (3rd-10th centile). There were no particular neonatal problems. Growth has continued below the 3rd centile while head circumference has remained on the 10th. Striking midfacial hypoplasia with a flattened nose, epicanthic folds, and smal hands were noted early on. She has had recurrent upper respiratory infections and otitis media associated with febrile convulsions on two occasions. Developmental milestones were delayed with sitting at 11 months, crawling at 15 months, and walking at 2 years. Investigations for delayed speech showed bilateral glue ear requiring grommets while audiograms indicated a possible additional high frequency hearing loss. Ophthalmological examination at 2 years of age was normal and investigations including renal, liver, and bone biochemical profiles, thyroid function, chromosomes, and urinary amino and organic acids have been normal. Examination at 4 years confirmed persisting midfacial hypoplasia with marked flattening of the nasal bridge and a small anteverted nose with a short columella (fig 

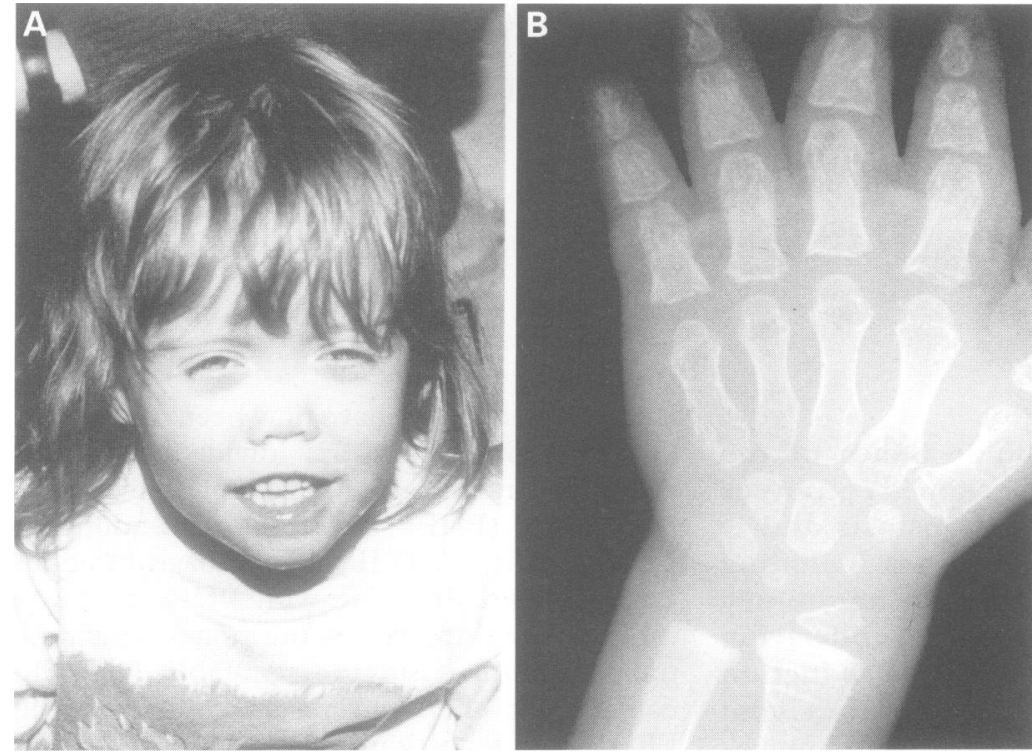

Figure 1 (A) Case 1 aged 4 years. (B) Plain radiograph of left hand of case 1 aged 19 months showing abnormally shaped and shortened metacarpals and phalanges with associated cone shaped epiphyses.

1A), striking generalised bony shortening in the hands with medial deviation of the left middle finger at the PIP joint, and small broad feet with relatively long halluces bilaterally. Hand $x$ rays showed generalised shortening of the tubular bones associated with cone shaped epiphyses (fig 1B).

All three sibs and both parents are of normal stature with no evidence of peripheral dysostosis or nasal hypoplasia.

CASE 2

This is the only child of healthy, unrelated parents and was delivered at 33 weeks' gestation by emergency caesarian section because of intrauterine growth retardation with oligohydramnios and breech presentation. At birth his weight was $1740 \mathrm{~g}$ (<3rd centile), length 44.5 $\mathrm{cm}$ ( $<3 \mathrm{rd}$ centile), and head circumference $30.1 \mathrm{~cm}(<3 \mathrm{rd}$ centile). He was noted to have a flat midface and short digits at birth as well as mild talipes and some limitation of elbow and knee extension, presumed secondary to oligohydramnios. Bilateral inguinal hernias were repaired. Hearing impairment was detected on routine screening and tympanograms indicated middle ear fluid for which grommets were inserted. Subsequent hearing tests have shown additional mild sensorineural hearing loss. Bone biochemistry profiles and thyroid function were normal at 8 months. Further investigation at 21 months showed normal serum calcium but slightly raised phosphate $(1.6 \mathrm{mmol} /$ 1), urea (11.2 mmol/1), and creatinine (73 $\mu \mathrm{mol} / \mathrm{l})$, and normal TRH, arginine stimulation, and synacthen tests. Renal ultrasound showed bilateral hydronephrosis and megaureters secondary to reflux on micturating cystography. Renal function has remained stably reduced on antibiotic prophylaxis. Head circumference, initially progressing along the 3 rd centile, increased to the 50 th centile when assessed at 2 years 7 months. CT scan of the head was normal. Currently, at 4 years 9 months, his height and weight are on the 3rd
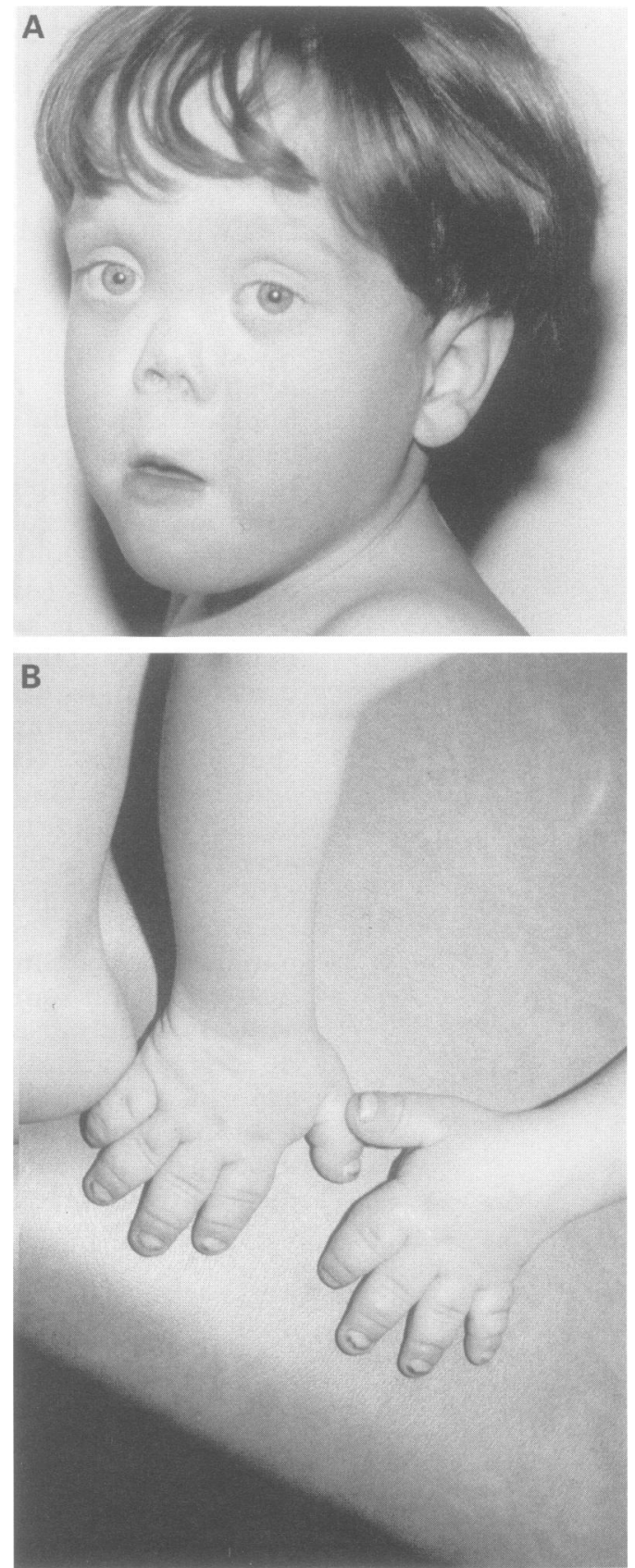

Figure 2 (A) Case 2 aged 4 years 10 months. (B) Hands of case 2 aged 4 years 10 months.

centile and OFC is on the 50th-75th centile. $\mathrm{He}$ has pronounced midfacial hypoplasia (fig 2A), a flat nasal bridge, small anteverted nose with a short columella, and striking shortening of all the tubular bones of the hands and feet (fig 2B). Developmental assessment has confirmed mild global developmental delay. His father's height is above the 97th centile and mother's between the 10 th and 25 th centiles.

MEASUREMENT OF GS $\alpha$ BIOACTIVITY

Five millilitres of venous blood in citrate anticoagulant was frozen on dry ice and stored at $-70^{\circ} \mathrm{C}$. Measurement of erythrocyte Gs $\alpha$ bioactivity was by cyc- reconstitution assay as described by Bourne et al (1983) and membranes were kindly provided by Dr C Van Dop. Purification of $\left[{ }^{32} \mathrm{P}\right]$ cAMP was as described by Salomon et al (1974) using Dowex Alumina chromatography and $\left[{ }^{3} \mathrm{H}\right] \mathrm{cAMP}$ to monitor 
Table 1 Results of erythrocyte Gsa bioactivity measurement expressed as a percentage of that of a concurrent sample from a healthy unrelated control

\begin{tabular}{lclll}
\hline & $\begin{array}{l}\text { Assay 1 } \\
(\%)\end{array}$ & $\begin{array}{l}\text { Assay 2 } \\
(\%)\end{array}$ & $\begin{array}{l}\text { Assay 3 } \\
(\%)\end{array}$ & Mean (\%) \\
\hline Case 1 & 93 & 103 & 107 & 101 \\
Case 2 & 126 & 101 & 122 & 116 \\
\hline
\end{tabular}

recovery. Sample eluates were counted in $8 \mathrm{ml}$ of scintillation fluid (Universol.ES) in a dual channel scintillation counter. Values are the mean of duplicates corrected for cAMP recovery and expressed as a percentage of the $\left[{ }^{32} \mathrm{P}\right]$ cAMP production in a concurrent sample from an unrelated healthy control.

DENATURING GRADIENT GEL ELECTROPHORESIS (DGGE) ANALYSIS OF EXONS 2-13 OF THE GS $\alpha$ GENE

Genomic DNA was extracted from peripheral lymphocytes using standard methods and screened by the mutational analysis procedure of DGGE as described elsewhere (Oude Luttikhuis et al, manuscript in preparation). Positive controls for exons 3-10 and 12 were included.

\section{Results}

GS $\alpha$ BIOACTIVITY

Both patients had normal erythrocyte Gs $\alpha$ bioactivity measured on three separate assays (table 1). Six unrelated subjects diagnosed with AHO (either PHP based on physical and endocrine findings or PPHP based on an AHO appearance without hormone resistance but either a first degree relative with PHP or the presence of cutaneous ossification) had Gs $\alpha$ bioactivity ranging from $53-73 \%$ (mean $62 \%$ ) of the same control sample over these assay runs.

GS $\alpha$ GENE MUTATION SCREENING

No sequence variations were detected on screening of exons 2-13 inclusive by DGGE (data not shown).

\section{Discussion}

We have shown normal Gs $\alpha$ bioactivity in two unrelated patients with acrodysostosis. Furthermore, we did not detect any sequence variation in a screen of 12 of the 13 exons and the adjacent splice sites of the Gs $\alpha$ gene assessed by DGGE, a procedure reported to approach

Table 2 Summary of clinical features of acrodysostosis and AHO

\begin{tabular}{|c|c|c|}
\hline & Acrodysostosis ${ }^{414}$ & $A H O^{79}$ \\
\hline Peripheral dysostosis & Severe, generalised & Variable, may be generalised \\
\hline Facial features & $\begin{array}{l}\text { Severe nasal and maxillary } \\
\text { hypoplasia }\end{array}$ & $\begin{array}{l}\text { Round face, mild midfacial } \\
\text { hypoplasia }\end{array}$ \\
\hline Short stature & Frequent, prenatal onset & Frequent, postnatal onset \\
\hline Mental retardation & Mild/moderate & Mild/moderate \\
\hline Hormone resistance & Absent $^{6}$ & PHP type I only \\
\hline Soft tissue ossification & Absent & $-50 \%$ \\
\hline Epiphyseal stippling & Neonatal period ${ }^{16}$ & Not reported \\
\hline Bone age & Frequently advanced & Frequently advanced \\
\hline $\begin{array}{l}\text { Vertebral interpedicular } \\
\text { distance }\end{array}$ & Loss of caudal widening & Loss of caudal widening \\
\hline Forearm brachymelia & Common & Not usually marked \\
\hline Relative 1 st ray hyperplasia & Common & Not usually marked \\
\hline Delayed dentition & Common & Common \\
\hline Otitis media/glue ear & Common & Common \\
\hline Inheritance & Probably $\mathrm{AD}^{18-21}$ & $\mathrm{AD}$ with ?parental origin effect ${ }^{22}{ }^{23}$ \\
\hline
\end{tabular}

$100 \%$ efficiency. ${ }^{12}$ The remaining exon 1 was not analysed because the very high GC nucleotide content makes it unamenable to screening by DGGE. In 17 unrelated patients with AHO in whom we have detected Gs $\alpha$ mutations, including those with exon 1 mutations detected by direct sequencing, all had reduced Gs $\alpha$ bioactivity (Oude Luttikhuis et al, manuscript in preparation). These results indicate that acrodysostosis in these patients is not caused by deactivating mutations in the Gs $\alpha$ gene, in contrast to AHO.

The clinical features of AHO and acrodysostosis are summarised in table 2. Although translated literally "acro"-dysostosis and "peripheral" dysostosis are synonymous, the latter is generally used in a descriptive sense. ${ }^{5}$ As such, peripheral dysostosis is a prominent feature of both acrodysostosis and AHO and may be a feature of other conditions including acromesomelic dysplasia, acromicric dysplasia, brachydactyly E, trichorhinophalangeal syndrome, and the Turner syndrome. In AHO, the resulting pattern of brachymetaphalangism may be as severe and generalised as in acrodysostosis, ${ }^{13}$ but is usually milder and more marked in the ulnar metacarpals and distal phalanges. When present, other features may be helpful in distinguishing the two. Endocrine disturbance $^{6}$ and cutaneous ossifications are not features of acrodysostosis. The short stature of acrodysostosis is often of prenatal onset $^{5}{ }^{14}$ and shortening of the extremities may be noted at birth, in contrast to AHO where birth weights and lengths are often normal and bony shortening of the hands and feet is frequently not manifest until 3 or 4 years of age $^{715}$ (unpublished data). Epiphyseal stippling, reported in the first few months of life in seven children with acrodysostosis, ${ }^{16}$ has not been reported in AHO, although this may reflect less obvious clinical indications for radiographs in infancy. Shortening of the nose and flattening of the nasal bridge is common in AHO but it is not usually striking, unlike acrodysostosis where there is marked nasal bony hypoplasia, anteversion of the nares, a deficient columella, prognathism, and flattening of the zygomas with prominence of the upper alveolar process causing a rather concave midface and open mouth appearance.

Occasional patients have been reported where the diagnoses of acrodysostosis and AHO were felt to coincide or to be indistinguishable. ${ }^{1017}$ Ablow et $a l^{10}$ reported two unrelated people of whom the first had clinical features, including soft tissue calcifications and resistance to $\mathrm{PTH}$, which leaves little doubt about a diagnosis of PHP. However, the nasal bridge was felt to be flattened to a greater extent than is usual in AHO. In contrast, the second patient had typical facial features of acrodysostosis without endocrine abnormalities or cutaneous ossification. In the report of Davies and Hughes, ${ }^{17}$ the absence of generalised severe brachymetaphalangism or marked nasal hypoplasia with deficient columella are more consistent with the PPHP form of AHO. In a further family, two sibs with typical features of acrodysostosis have been described 
whose mother had normal stature and intelligence, mild nasal hypoplasia, and variable bony shortening in the hands and feet, closely resembling PPHP phenotypically. ${ }^{18}$ She may represent a somatic mosaic for acrodysostosis. These families illustrate the difficulties that may occur differentiating these diagnoses clinically.

The two patients we report closely resemble the original phenotypic descriptions of acrodysostosis. While at a molecular level there may be heterogeneity underlying the acrodysostosis phenotype, our findings of normal Gs $\alpha$ bioactivity in conjunction with the negative Gs $\alpha$ mutation screen support the contention that at least in a proportion of patients this condition is aetiologically distinct from $\mathrm{AHO}$.

LCW was an MRC (UK) Clinical Training Fellow during part of this work. RCT acknowledges the Research Trust for Metaof this work. RCT acknowledges the Research Trust for Meta-
bolic Diseases of Childhood for support. We thank both families for their cooperation.

1 Brailsford JF. The radiology of bones and joints. Baltimore: William \& Wilkins, 1948:33.

2 Cohen P, van Creveld S. Peripheral dysostosis. $\mathrm{Br} \mathcal{F}$ Radio 1963;36:761-5

3 Giedion A. Die Periphere Dysostose (PD): Ein sammelbegriff. Fortschr Roentgenstr 1969;110:507-24

4 Maroteaux P, Malamut G. L'acrodysostose. Presse Med 1968;76:2189-92.

5 Robinow M, Pfeiffer RA, Gorlin RJ, et al. Acrodysostosis: a syndrome of peripheral dysostosis, nasal hypoplasia, and mental retardation. Am $\mathcal{F}$ Dis Child 1971;121:195-203.

6 Garces LY, Blank E, Drash AL, Kenny FM. Peripheral dysostosis: investigation of metabolic and endocrine functions. F Pediatr 1969;74:730-7.

7 Fitch N. Albright's hereditary osteodystrophy: a review. $A m$ f Med Genet 1982;11:11-29.
8 Spiegel AM. Albright's hereditary osteodystrophy and defective G proteins. N Engl F Med 1990;322:1461-2.

9 Wilson LC, Trembath RC. Albright's hereditary osteodystrophy. F Med Genet 1994;31:779-84.

10 Ablow RC, Hsia YE, Brandt IK. Acrodysostosis coinciding with pseudohypoparathyroidism and pseudopseudohypoparathyroidism. AfR 1977;128:95-9.

11 Gorlin RJ, Cohen M Jr, Levin LS. Syndromes of the head and neck. Oxford:Oxford University Press, 1990:139-43.

12 Beck YS, Kwitek AE, Cogen PH, et al. A denaturing gradient gel electrophoresis assay for sensitive detection of p53 mutations. Hum Genet 1993;91:25-30.

13 Poznanski AK, Werder EA, Giedion A. The pattern of shortening of the bones of the hand in PHP and PPHP comparison with brachydactyly E, Turner syndrome, and acrodysostosis. Radiology 1977;123:707-18.

14 Butler MG, Rames LJ, Wadlington WB. Acrodysostosis: report of a 13-year-old boy with review of literature and metacarpophalangeal pattern profile analysis. $\mathrm{Am} \mathcal{f} \mathrm{Med}$ Genet 1988;30:971-80.

15 de Wijn EM, Steendijk R. Growth and maturation in pseudo-hypoparathyroidism; a longitudinal study in 5 patients. Acta Endocrinol 1982;101:223-6.

16 Viljoen D, Beighton P. Epiphyseal stippling in acrodysostosis. Am f Med Genet 1991;38:43-5.

17 Davies SJ, Hughes HE. Familial acrodysostosis: can it be distinguished from Albright's hereditary osteodystrophy? Clin Dysmorphol 1992;1:207-15.

18 Niikawa N, Matsuda I, Ohsawa T, Kajii T. Familial occurrence of a syndrome with mental retardation, nasal hypoplasia, peripheral dysostosis, and blue eyes in Japanese siblings. Hum Genet 1978;42:227-32.

19 Steiner RD, Pagon RA. Autosomal dominant transmission of acrodysostosis. Clin Dysmorphol 1992;1:201-6.

20 Hernandez RM, Miranda A, Kofman-Alfaro S. Acrodysostosis in two generations: an autosomal dominant syndrome. Clin Genet 1991;39:376-82.

21 Frey VG, Martin J, Dietel K. Die akrodysostose - ein autosomal - dominant vererbte periphere dysplasie. Kinderartz Prax 1982;3:149-53.

22 Wilson LC, Oude Luttikhuis MEM, Clayton PT, Frase WD, Trembath RC. Parental origin of Gs $\alpha$ mutations in Albright's hereditary osteodystrophy. 7 Med Genet 1994;31: 835-9.

23 Davies SJ, Hughes HE. Imprinting in Albright's hereditary osteodystrophy. 7 Med Genet 1993;30:101-3. 